\title{
Spin accumulation in the extrinsic spin Hall effect
}

\author{
Wang-Kong Tse, ${ }^{1}$ J. Fabian, ${ }^{2}$ I. Žutić, ${ }^{1,3}$ and S. Das Sarma ${ }^{1}$ \\ ${ }^{1}$ Condensed Matter Theory Center, Department of Physics, University of Maryland at College Park, College Park, \\ Maryland 20742-4111, USA \\ ${ }^{2}$ Institute for Theoretical Physics, University of Regensburg, 93040 Regensburg, Germany \\ ${ }^{3}$ Center for Computational Materials Science, Naval Research Laboratory, Washington, D.C. 20735, USA
}

(Received 2 August 2005; revised manuscript received 3 October 2005; published 7 December 2005)

\begin{abstract}
The drift-diffusion formalism for spin-polarized carrier transport in semiconductors is generalized to include spin-orbit coupling. The theory is applied to treat the extrinsic spin Hall effect using realistic boundary conditions. It is shown that carrier and spin-diffusion lengths are modified by the presence of spin-orbit coupling and that spin accumulation due to the extrinsic spin Hall effect is strongly and qualitatively influenced by boundary conditions. Analytical formulas for the spin-dependent carrier recombination rates and inhomogeneous spin densities and currents are presented.
\end{abstract}

DOI: 10.1103/PhysRevB.72.241303

PACS number(s): 72.25.Dc, 72.25.Hg, 75.80.+q

In the presence of spin-orbit (S-O) coupling, either due to impurities or due to host lattice ions, carriers of opposite spins tend to scatter into opposite directions. With an electric field induced (longitudinal) motion under bias, the S-O scattering results in a transverse spin current and spin accumulation, as predicted by D'yakonov and Perel', ${ }^{1,2}$ and later revisited by others. ${ }^{3}$ This effect, which is now called the extrinsic spin Hall effect (SHE), ${ }^{4}$ has been recently demonstrated experimentally in $n$-GaAs and $n$-InGaAs thin films ${ }^{5}$ and in two-dimensional electron gas confined within (110) AlGaAs quantum wells. ${ }^{6}$ The signature of the effect is opposite spin accumulation at the edges of the sample, with spin polarization perpendicular to the transport plane.

This paper has two goals. First, we present a formalism for carrier drift and diffusion in inhomogeneous spinpolarized semiconductors in the presence of S-O coupling and spin-dependent band-to-band electron-hole recombination. The formalism, which is a generalization of a previous spin and charge drift-diffusion theory, ${ }^{7,8}$ applies to both unipolar and bipolar cases, the former being a subclass of the latter. Second, we apply the formalism to explain the main qualitative features of spin accumulation in the extrinsic SHE in the optical orientation experiment, for two different boundary conditions: (a) uniform generation of electron-hole pairs, and (b) edge generation of nonequilibrium electrons. In both cases spin accumulation throughout the sample is calculated analytically. We find that $\mathrm{S}-\mathrm{O}$ interaction modifies the carrier and spin diffusion lengths and that the spin accumulation profile depends, qualitatively, on the specific boundary conditions, implying that interpretation of extrinsic SHE requires detailed case-by-case considerations for specific experimental and sample geometries.

Consider spin-polarized transport in an inhomogeneous nonmagnetic semiconductor in the presence of electric field E. If S-O coupling is present, causing skew scattering and side jump, the phenomenological expression for the carrier ( $c=n$ for electrons and $c=p$ for holes) charge current density in the $i$ th direction is readily obtained by generalizing the Dyakonov-Perel' prediction ${ }^{1,2}$

$$
J_{c \lambda, i}=q \mu_{c \lambda} c_{\lambda} E_{i} \pm q D_{c \lambda} \partial_{i} c_{\lambda}+q \lambda \nu_{c \lambda} c_{\lambda} \epsilon_{i j z} E_{j}+q \lambda \delta_{c \lambda} \epsilon_{i j z} \partial_{j} c_{\lambda}
$$

Here the upper (lower) sign is for electrons (holes) and $\lambda$ is the spin index; $q$ is the proton charge. The first two terms are conventional (longitudinal) carrier drift and diffusion, respectively, with $\mu$ and $D$ denoting the spin-dependent mobility and diffusivity. The third (fourth) term represents the effects of skew spin-orbit scattering and side jump on drift (diffusion). The effects of the scattering are in the transverse direction to $\mathbf{E}$ and are opposite for spin-up and spin-down carriers. (Holes are treated here as spin doublets, which is appropriate in low-dimensional structures with heavy and light hole band splitting; otherwise hole spin does not matter, as we will argue below). The corresponding transport parameters are transverse mobility $\nu$ and transverse diffusivity $\delta$; they are proportional to the $\mathrm{S}-\mathrm{O}$ coupling strength.

It is more illuminating to introduce the charge, $J_{c}=J_{\uparrow}+J_{\downarrow}$, and spin, $J_{s}=J_{\uparrow}-J_{\downarrow}$, currents. In terms of carrier $\left(c=c_{\uparrow}+c_{\downarrow}\right)$ and spin $\left(s_{c}=c_{\uparrow}-c_{\downarrow}\right)$ densities, the currents are

$$
\begin{aligned}
J_{c, i}= & q\left(\mu_{c} c+\mu_{s c} s_{c}\right) E_{i} \pm q\left(D_{c} \partial_{i} c+D_{s c} \partial_{i} s_{c}\right) \\
& +q \epsilon_{i j z} E_{j}\left(\nu_{c} s_{c}+\nu_{s c} c\right)+q \epsilon_{i j z}\left(\delta_{c} \partial_{j} s_{c}+\delta_{s c} \partial_{j} c\right), \\
J_{s c, i}= & q\left(\mu_{s c} c+\mu_{c} s_{c}\right) E_{i} \pm q\left(D_{c} \partial_{i} s_{c}+D_{s c} \partial_{i} c\right) \\
& +q \epsilon_{i j z} E_{j}\left(\nu_{c} c+\nu_{s c} s_{c}\right)+q \epsilon_{i j z}\left(\delta_{c} \partial_{j} c+\delta_{s c} \partial_{j} s_{c}\right) .
\end{aligned}
$$

The transverse carrier charge and spin mobilities are given respectively by $\nu_{c}=\left(\nu_{c \uparrow}+\nu_{c \downarrow}\right) / 2$ and $\nu_{s c}=\left(\nu_{c \uparrow}-\nu_{c \downarrow}\right) / 2$, while the transverse carrier charge and spin diffusivities are $\delta_{c}=\left(\delta_{c \uparrow}+\delta_{c \downarrow}\right) / 2$ and $\delta_{s c}=\left(\delta_{c \uparrow}-\delta_{c \downarrow}\right) / 2$. The corresponding longitudinal quantities are defined similarly.

Equations (2) and (3) succinctly describe the appearance of the transverse spin drift and diffusion in the presence of longitudinal charge transport, which is the essence of the extrinsic SHE. If the longitudinal current is spin polarized, the above equations describe the anomalous Hall effect ${ }^{9}$ and the appearance of the transverse Hall voltage. 
To further develop the formalism, we need to include electron-hole recombination and spin relaxation. In the presence of S-O coupling, spin-dependent selection rules ${ }^{10}$ for band-to-band transitions need to be considered. In general, the continuity equation reads

$$
\begin{aligned}
\pm \partial_{i} J_{c \lambda, i} / q= & B_{1}\left(c_{\lambda} \bar{c}_{\lambda}-c_{0 \lambda} \bar{c}_{0 \lambda}\right)+B_{2}\left(c_{\lambda} \bar{c}_{\bar{\lambda}}-c_{0 \lambda} \bar{c}_{0 \bar{\lambda}}\right) \\
& +\left(c_{\lambda}-c_{\bar{\lambda}}\right) / 2 T_{1 c} .
\end{aligned}
$$

Here $\bar{c}$ is $p(n)$ if $c$ is $n(p), c_{0}$ is the equilibrium carrier density, and $T_{1 c}$ is the $T_{1}$ time for spin flipping.

The spin-preserving recombination rate coefficient $B_{1}$, as well as the spin-flip coefficient $B_{2}$, can be calculated by generalizing the unpolarized case. ${ }^{11,12}$ The valence band of zincblende semiconductors consists of three subbands: heavy hole, light hole, and split-off hole bands. We neglect the split-off band as the energy splitting $\Delta \gg k_{B} T$ at temperatures $T$ lower than or around room temperature. By explicitly taking into account the angular momentum of the heavy hole and light hole states, and using the optical selection rules for the states, ${ }^{10}$ we arrive at the following expressions for spinconserving and spin-flip recombination constants:

$$
\begin{gathered}
B_{1}=C \frac{\left[m_{h} /\left(m_{c}+m_{h}\right)\right]^{3 / 2}+\frac{2}{3}\left[m_{l} /\left(m_{c}+m_{l}\right)\right]^{3 / 2}}{m_{h}^{3 / 2}+m_{l}^{3 / 2}}, \\
B_{2}=\frac{1}{3} C \frac{\left[m_{h} /\left(m_{c}+m_{h}\right)\right]^{3 / 2}}{m_{h}^{3 / 2}+m_{l}^{3 / 2}},
\end{gathered}
$$

where $C$ depends on the "maximum" electron energy $\hbar \omega_{\max }=\epsilon_{g}+k_{B} T / 2\left(\epsilon_{g}\right.$ is the energy gap) and temperature,

$$
C\left(\hbar \omega_{\max }, T\right)=\frac{4 e^{2}}{\hbar^{2} m^{2} c^{3}}\left(\frac{2 \pi \hbar^{2}}{k_{B} T}\right)^{3 / 2} P^{2} n_{r} \hbar \omega .
$$

Here $P$ is the momentum matrix element for optical transitions, $n_{r}$ is the refractive index, $m_{c}\left(m_{h}\right.$ and $\left.m_{l}\right)$ is the band mass of electrons (heavy holes and light holes).

Equations (1) and (4), together with Poisson's equation form a closed set of nonlinear equations whose solution determines charge and spin densities and currents in a semiconductor with S-O scattering included. In general, these equations need to be solved numerically for specific cases of interest. Our next goal is to introduce qualitative features of spin accumulation in two cases of experimental interest that allow analytical solutions and form a starting point to discuss the concepts and issues to be encountered in more complex situations involving SHE and S-O coupling effects in transport. We consider a $p$-type semiconductor with nondegenerate electron (minority) density induced optically. The resulting spin accumulation via extrinsic SHE can be deduced in a manner similar to spin orientation experiments. We assume that the injected electron density is well below the donor density. Further simplification follows from the fact that mobilities and diffusivities are spin independent in the nondegenerate regime. ${ }^{7}$ Finally, we assume unpolarized holes since hole spin relaxation in zinc-blende semiconductors is extremely fast. ${ }^{13}$ The only carriers of interest are then spinpolarized electrons.
Using the above assumptions, Eqs. (2)-(4), give the driftdiffusion equations for electron spin and carrier density

$$
\begin{aligned}
\nabla^{2} s & +\left(q / k_{B} T\right)(s \nabla \cdot \mathbf{E}+\mathbf{E} \cdot \nabla s)+\left(\zeta q / k_{B} T\right)(\nabla n \times \mathbf{E})_{z} \\
& +\left(w p+1 / T_{1 n}\right) s=0, \\
\nabla^{2} n & +\left(q / k_{B} T\right)(n \nabla \cdot \mathbf{E}+\mathbf{E} \cdot \nabla n)+\left(\zeta q / k_{B} T\right)(\nabla s \times \mathbf{E})_{z} \\
& +w\left(n p-n_{0} p_{0}\right)=0,
\end{aligned}
$$

where $w=\left(B_{1}+B_{2}\right) / 2$ and $\zeta=\nu_{n} / \mu_{n}=\delta_{n} / D_{n}$ characterizes the $\mathrm{S}-\mathrm{O}$ coupling strength, $n_{0}, p_{0}$ are the equilibrium electron and hole densities. The spin quantization axis is taken to be $z$. We take $\mathbf{E}=E \hat{\mathbf{y}}$ and consider $x$ to be the transverse direction, the slab boundaries being at $x=0$ and $x=a$, so that all the quantities of interest will have $x$ dependence only. Denoting the electron recombination time as $\tau_{n}=1 /\left(w N_{a}\right)$, where $N_{a}$ is the acceptor density, and spin relaxation time as $\tau_{s}$ $=1 /\left(\tau_{n}^{-1}+T_{1 n}^{-1}\right)$, Eqs. (8) and (9) become

$$
\begin{gathered}
\ddot{s}+\left(\zeta q E / k_{B} T\right) \dot{n}-s / L_{s}^{2}=0, \\
\ddot{n}+\left(\zeta q E / k_{B} T\right) \dot{s}-\left(n-n_{0}\right) / L_{n}^{2}=0,
\end{gathered}
$$

where derivatives with respect to $x$ is denoted by overdots, the longitudinal spin and charge diffusion lengths are defined as $L_{s}=\sqrt{D_{s} \tau_{s}}$ and $L_{n}=\sqrt{D_{n} \tau_{n}}$, respectively. In deriving the above equations we have neglected terms of order $s^{2}, s \delta n$, and $\delta n^{2}$ relative to $\delta n=n-n_{0} .{ }^{14}$ Spin-charge coupling in Eqs. (10) and (11) is apparent through the first-order derivatives of spin and charge densities.

We first solve the transverse spin and carrier diffusion for the case of a uniformly illuminated slab (boundary condition BC I), with electron-hole spin-unpolarized generation rate $G$. We assume that carrier recombination at the edges is not significant so that it is reasonable to impose a uniform (here zero) electron transverse current: $J_{n x}(x)=0$. As for spin current, we take $J_{s}(x=0)=J_{s}(x=a)=0$, implying that spin-flip scattering at the edges is moderate. The solution to the driftdiffusion Eq. (10) in the presence of S-O scattering is

$$
s(x)=\zeta \frac{q E L_{s}}{k_{B} T} G \tau_{n} \operatorname{sech}\left(a / 2 L_{s}\right) \sinh (x / L-a / 2 L) .
$$

The spin-polarization profile is given by $\alpha(x)=s(x) / G \tau_{n}$, since $G \tau_{n} \gg n_{0}$ is the average electron density generated in steady-state conditions under illumination. This simple solution demonstrates the essential physics behind spin accumulation in extrinsic SHE: (i) Spin accumulation increases linearly with $E$, with possible slight electric field modulation due to the dependence of $L_{s}=L_{s}(E)$ (not discussed here). (ii) The magnitude of spin polarization is proportional to the strength of the S-O scattering as well as to the ratio of the voltage drop over $\min \left(L_{s}, a\right)$ and thermal energy. (iii) For $a \ll L_{s}$ the accumulation at the edges is linearly proportional to $a$, while for $a \gg L_{s}$, the accumulation is independent of $a$. (iv) While $\alpha(x) \sim x$ for $a \ll L_{s}$, spin accumulation is significant only within the spin diffusion length from the edges when $a \gg L_{s}$.

A question now arises: How universal (i.e., independent of boundary conditions) is the qualitative behavior discussed 
above? To answer this question we introduce different boundary conditions (BC II), describing the physics of carrier injection at $x=0$ and $x=a$, while assuming vanishing spin currents at the edges: $n(x=0)=n_{1}, n(x=a)=n_{2} ; J_{s, x}(x=0)$ $=J_{s, x}(x=a)=0$. These conditions mimic the case of a $p$-doped base in a pnp spin-polarized transistor, ${ }^{15}$ where the electron injection level can be controlled by the biases to the emitter and collector. In the case discussed here the longitudinal current would flow perpendicular to the transistor current, which would thus be spin polarized due to the extrinsic SHE. The vanishing boundary conditions for $J_{s, x}$ reduce to $\dot{s}(x=0)=-\zeta q E n_{1} / k_{B} T$ and $\dot{s}(x=a)=-\zeta q E n_{2} / k_{B} T$. Solving Eqs. (10) and (11) with the above boundary conditions gives the spin and electron densities inside the slab

$$
s(x)=\frac{\zeta q E}{k_{B} T\left(L_{1}^{-2}-L_{2}^{-2}\right)}\left[\frac{1}{L_{1}} C_{1}(x)-\frac{1}{L_{2}} C_{2}(x)\right],
$$

and

$$
\begin{aligned}
n(x)-n_{0}= & \frac{1}{\left(L_{1}^{-2}-L_{2}^{-2}\right)}\left[\left(L_{1}^{-2}-L_{s}^{-2}\right) S_{1}(x)\right. \\
& \left.-\left(L_{2}^{-2}-L_{s}^{-2}\right) S_{2}(x)\right],
\end{aligned}
$$

where for convenience we have defined the functions

$$
\begin{gathered}
C_{1,2}=\left[n_{1} \cosh \left(\frac{a-x}{L_{1,2}}\right)-n_{2} \cosh \left(\frac{x}{L_{1,2}}\right)\right] / \sinh \left(\frac{a}{L_{1,2}}\right), \\
S_{1,2}=\left[n_{1} \sinh \left(\frac{a-x}{L_{1,2}}\right)+n_{2} \sinh \left(\frac{x}{L_{1,2}}\right)\right] / \sinh \left(\frac{a}{L_{1,2}}\right) .
\end{gathered}
$$

Here we introduce new transverse spin-charge diffusion lengths, $L_{1}$ and $L_{2}$,

$$
L_{1,2}^{-2}=\gamma \pm \sqrt{\gamma^{2}-\left(1 / L_{s} L_{n}\right)^{2}},
$$

where

$$
\gamma=(1 / 2)\left[L_{s}^{-2}+L_{n}^{-2}+\left(\zeta q E / k_{B} T\right)^{2}\right] .
$$

There is a critical value of the field that separates the regimes of strong and weak spin-charge coupling-Eqs. (10) and (11) reduce to the ordinary spin and charge diffusion equations when $E \ll E_{s}, \quad E_{n}$, where $E_{s}=k_{B} T /\left(\zeta q L_{s}\right)$ and $E_{n}$ $=k_{B} T /\left(\zeta q L_{n}\right)$ are the values of the critical fields with respect to spin and charge diffusion. In this case $\gamma \simeq\left(L_{s}^{-2}+L_{n}^{-2}\right) / 2$ and $L_{1,2} \simeq L_{s, n}$. When $E \gtrsim E_{s}, E_{n}, \gamma$ becomes dependent on the electric field and it is in this regime the spin-field relation deviates from linearity.

For quantitative understanding we take our semiconductor to be $\mathrm{GaAs}$ at room temperature, ${ }^{16}$ with doping density $N_{a}=3 \times 10^{15} \mathrm{~cm}^{-3}$, and transverse size of $a=6 \mu \mathrm{m}$ (which is much greater than $L_{s}$ ). The S-O scattering strength is taken to be $\zeta=10^{-4}$, reflecting weak $\mathrm{S}-\mathrm{O}$ coupling in GaAs. Finally, the boundary conditions for electron density are $n(0)=2 \times 10^{13} / \mathrm{cm}^{3}$ and $n(a)=5 \times 10^{13} / \mathrm{cm}^{3}$. Figure 1 shows the profiles of spin and electron densities, as well as spin polarization $\alpha=s / n$, for several values of $E$. In contrast to the

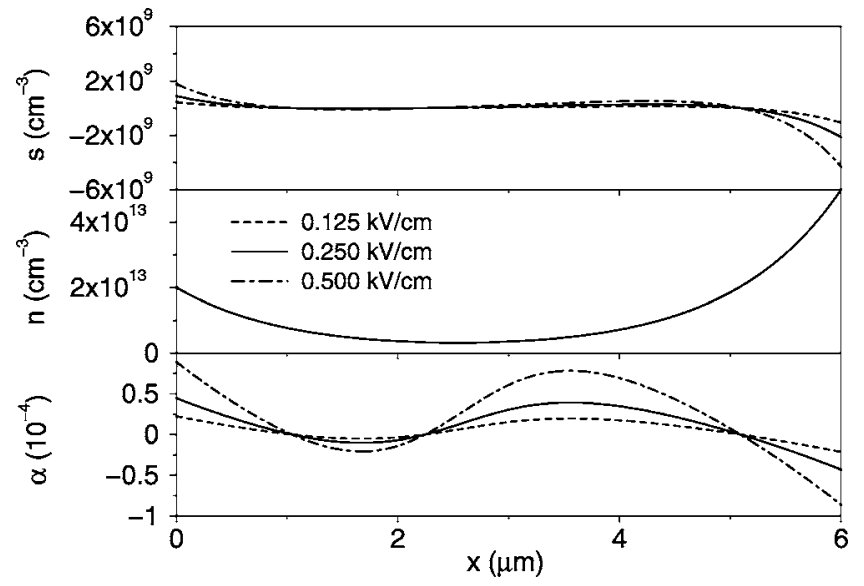

FIG. 1. Calculated spin density $s$, electron density $n$, and polarization $\alpha$ for BC II. We have used $\zeta=10^{-4}$ and applied electric field strengths $0.125,0.250$, and $0.500 \mathrm{kV} / \mathrm{cm}$. While a signature of the extrinsic SHE is the opposite spin accumulation at the edges of the sample, spin accumulation is large also inside.

purely diffusive behavior exhibited by $n$, spin density along the slab exhibits weakly oscillatory behavior. Both spin density and spin polarization attain maximum magnitudes at the edges. (We find that spin polarization is enhanced by a decade when considering the extrinsic SHE at $77 \mathrm{~K}$.) What is interesting is, unlike in BC I [conclusion (iv)], that spin accumulation here is significant throughout the sample, not just within $L_{s}$ from the edges. This oscillatory pattern is due to the existence of spin-charge coupling that couples the spin and charge diffusion lengths together into two spin-charge diffusion lengths Eqs. (17) and (18), so that the net spindensity profile Eq. (13) can be thought of as the interference between two spin-density profiles, each having a characteristic spin-charge diffusion length $L_{1}$ or $L_{2}$. Since, as a result of spin Hall effect, each of these terms changes sign near the boundaries, they interfere destructively and constructively along the slab giving rise to the oscillatory pattern in Fig. 1 (similar arguments apply for the electron density, but since each of these terms is always positive, they add up constructively). We note that similar oscillatory behavior is also observed in a number of recent papers ${ }^{17}$ on the intrinsic SHE, and in a recent experiment. ${ }^{6}$ In the presence of magnetic field

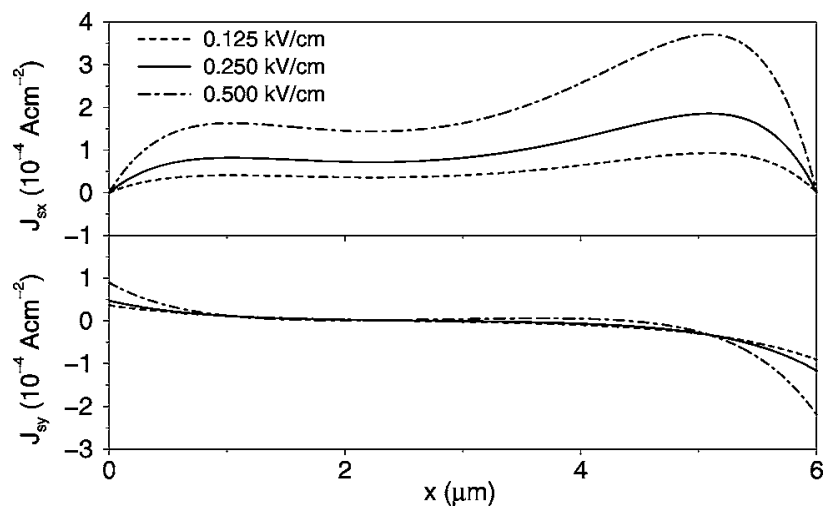

FIG. 2. Calculated spin current $J_{s}$ in $x$ (top) and $y$ (bottom) directions for BC II and parameters as in Fig. 1. 


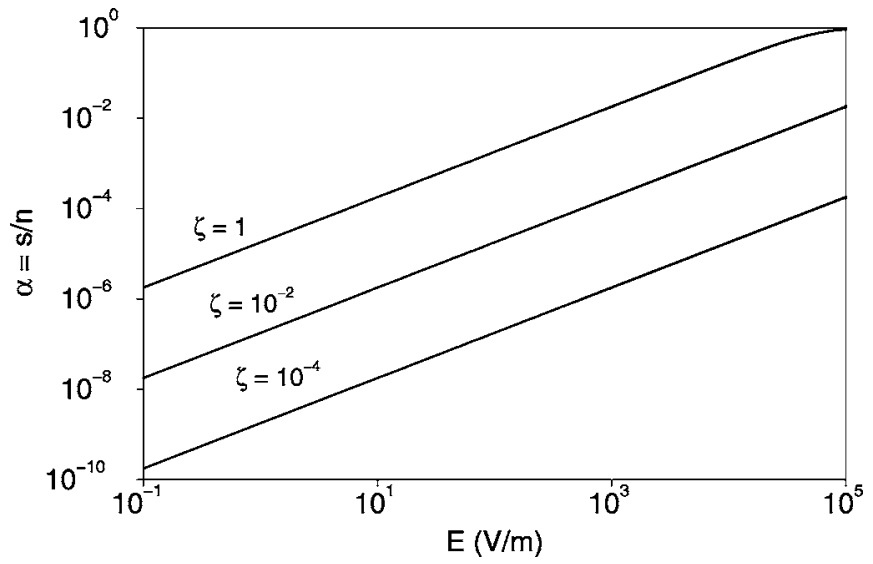

FIG. 3. Calculated spin polarization $\alpha$ at $x=0$ as a function of electric field $E$ for BC II and $\zeta=10^{-4}, 10^{-2}$, and 1 . Only in the last (unphysical) case $\alpha$ starts to saturate for large $E$, as the dependence of $L_{1}$ and $L_{2}$ on $E$ sets in.

(externally applied or effective field due to, e.g., Rashba S-O coupling), the oscillatory behavior comes not only from the coupling of spin and charge but also the coupling in between the different components of the spin. ${ }^{17}$ Now spin current is not conserved and flows inside the sample, being restricted to zero by our choice of boundary conditions (see Fig. 2). From Eq. (3) we obtain the spin current density in the $x$ direction

$$
J_{s, x}(x)=-\frac{q \nu_{n} E}{\left(L_{1}^{-2}-L_{2}^{-2}\right) L_{s}^{2}}\left[S_{1}(x)-S_{2}(x)\right]
$$

and in the $y$ direction

$$
\begin{aligned}
J_{s, y}(x)= & \frac{1}{\left(L_{1}^{-2}-L_{2}^{-2}\right)}\left\{\left[q \nu_{n} E^{2} / k_{B} T+\delta_{n}\left(L_{1}^{-2}-L_{s}^{-2}\right)\right] C_{1}(x) / L_{1}\right. \\
& \left.-\left[q \nu_{n} E^{2} / k_{B} T+\delta_{n}\left(L_{2}^{-2}-L_{s}^{-2}\right)\right] C_{2}(x) / L_{2}\right\} .
\end{aligned}
$$

Spin current in the $x$ direction flows in the direction of the spin gradient, while the $y$ component changes sign inside the slab, reflecting the fact that spin-up and spin-down electrons are deflected into opposite directions due to S-O scattering by impurities. Finally, we wish to see at what value of the electric field spin-charge diffusion lengths $L_{1,2}$ will be modified and induce nonlinear behavior in $\alpha$. Figure 3 shows $\alpha(E)$ for different $\zeta$. Spin polarization varies linearly with $E$, except at electric field as large as $10^{5} \mathrm{kV} / \mathrm{cm}$ and $\mathrm{S}-\mathrm{O}$ coupling $\zeta \approx 1$, a clearly unphysical case considered here only to illustrate the scope of linear behavior.

In conclusion, we have presented a drift-diffusion formalism which takes into account S-O scattering and spindependent carrier recombination. We have calculated spin accumulation in the extrinsic spin Hall regime and introduced S-O dependent spin-charge diffusion lengths. We have found that spin accumulation is strongly influenced by boundary conditions and thus by specific spintronic device design. Our theory is applicable to any complex device setting in which extrinsic spin Hall effect is expected to play a role. We expect similar strong dependence of the "intrinsic" SHE on boundary conditions too, making it difficult to distinguish intrinsic and extrinsic SHE in general.

This work was supported by the US-ONR and NSF.
${ }^{1}$ M. I. D'yakonov and V. I. Perel, JETP Lett. 13, 467 (1971).

${ }^{2}$ M. I. D'yakonov and V. I. Perel, Phys. Lett. 35A, 459 (1971).

${ }^{3}$ J. E. Hirsch, Phys. Rev. Lett. 83, 1834 (1999); S. Zhang, ibid. 85, 393 (2000); H.-A. Engel, et al., ibid. 95, 166605 (2005); W.-K. Tse and S. Das Sarma, cond-mat/0507149 (unpublished); E. M. Hankiewicz and G. Vignale, cond-mat/0507228 (unpublished).

${ }^{4}$ The term "extrinsic," to be distinguished from the "intrinsic SHE," was used in J. Sinova et al., Phys. Rev. Lett. 92, 126603 (2004).

${ }^{5}$ Y. K. Kato et al. Science 306, 1910 (2004).

${ }^{6}$ V. Sih et al., Nat. Phys. 1, 31 (2005).

${ }^{7}$ I. Žutić et al., Phys. Rev. Lett. 88, 066603 (2002).

${ }^{8}$ J. Fabian et al., Phys. Rev. B 66, 165301 (2002).

${ }^{9}$ R. Karplus and J. M. Luttinger, Phys. Rev. 95, 1154 (1954); P. Noziéres and C. Lewiner, J. Phys. (Paris) 34, 901 (1973).

${ }^{10}$ M. I. D’yakonov and V. I. Perel, in Optical Orientation (Elsevier Science, New York, 1984).

${ }^{11} \mathrm{H}$. Barry Bebb and E. W. Williams, in Semiconductors and Semi- metals (Academic Press, New York, 1972), Vol. 8.

${ }^{12}$ G. Lasher and F. Stern, Phys. Rev. 133, A553 (1964).

${ }^{13}$ I. Žutić et al., Rev. Mod. Phys. 76, 323 (2004).

${ }^{14}$ R. A. Smith, Semiconductors (Cambridge University Press, New York, 1978).

${ }^{15}$ J. Fabian, et al. cond-mat/0211639 (unpublished); J. Fabian, I. Žutić, Phys. Rev. B 69, 115314 (2004).

${ }^{16}$ For $T=300 \mathrm{~K}$, the transport parameters for the minority electron are taken as (Ref. 7): diffusivity $D_{n}=103.6 \mathrm{~cm}^{2} \mathrm{~s}^{-1}$, mobility $\mu_{n}=4000 \mathrm{~cm}^{2} \mathrm{~V}^{-1} \mathrm{~s}^{-1}$, recombination rate $w=(1 / 3)$ $\times 10^{-5} \mathrm{~cm}^{3} \mathrm{~s}^{-1}$ and spin-flip time $T_{1}=0.2 \mathrm{~ns}$. From these the calculated values of electron-hole recombination and spin diffusion lengths are, respectively, $L_{n}=1 \mu \mathrm{m}$ and $L_{s}=0.8 \mu \mathrm{m}$. The intrinsic concentration is $n_{i}=1.8 \times 10^{6} \mathrm{~cm}^{-3}$.

${ }^{17}$ A. A. Burkov et al. Phys. Rev. B 70155308 (2004); E. I. Rashba, cond-mat/0507007, Physica E (to be published); I. Adagideli, cond-mat/0506531, Phys. Rev. Lett. (to be published); B. K. Nikolic et al., Phys. Rev. Lett. 95, 046601 (2005). 